\title{
Cross-Layer Duty Cycle Scheduling with Data Aggregation Routing in Wireless Sensor Networks
}

\author{
Yean-Fu Wen ${ }^{1,2}$ and Frank Yeong-Sung Lin ${ }^{1}$ \\ ${ }^{1}$ National Taiwan University, Taiwan(R.O.C.) \\ ${ }^{2}$ China University of Technology, Taiwan(R.O.C.) \\ $\{$ d89002, yslin\}@im.ntu.edu.tw
}

\begin{abstract}
Well-scheduled communications, in conjunction with the aggregation of data reduce the energy waste on idle listening and redundant transmissions. In addition, the adjustable radii and the number of retransmissions are considered to reduce the energy consumption. Thus, to see that the total energy consumption is minimized, we propose a mathematical model that constructs a data aggregation tree and schedules the activities of all sensors under adjustable radii and collision avoidance conditions. As the data aggregation tree has been proven to be a NPcomplete problem, we adopt a LR method to determine a near-optimal solution and furthermore verify whether the proposed LR-based algorithm, LRA, achieves energy efficiency and ensures the latency within a reasonable range. The experiments show the proposed algorithm outperforms other general routing algorithms, such as SPT, CNS, and GIT algorithms. It improves energy conservation, which it does up to $9.1 \%$ over GIT. More specifically, it also improves energy conservation up to $65 \%$ over scheduling algorithms, such as S-MAC and T-MAC.
\end{abstract}

\section{Introduction}

The network lifetime of a wireless sensor network (WSN), the time before communication of the environmental information is interrupted because of depleted batteries is dependent on battery capacity and energy consumption efficiency, and has become an essential issue, as we can read in 22 [4] [7] 11 [13 [14] 16] 22]. Therefore, we seek to prolong network lifetime from the physical layer up to application layer, and do so with a focus on (i) the data aggregation routing; (ii) duty cycle scheduling; (iii) adjustable radii; and (iv) collision avoidance.

The data aggregation capability has been put forward as a particularly useful function for routing in terms of energy consumption in WSNs. Some literature [2] [11] 12] 23] 22 has been shown the in-network processing can save much energy. The construction of this type of data aggregation tree (i.e., a kind of reversemulticast tree which is also a Steiner tree) has been proven to be NP-complete 10, which signifies that general algorithms cannot provide optimal solution to the problem. Krishnamachari et al. [12] devised three aggregation heuristics, 
namely, the Shortest Paths Tree (SPT), Center at Nearest Source (CNS), and the Greedy Incremental Tree (GIT) to sub-optimally solve the problem. In our experimental results, we will compare the performance with these heuristics.

In addition to data aggregation, conservation of energy is accomplished by duty cycle, the reduction of idle listening that is also the most energy wasteful process in MAC protocol. Some researchers [8] [15] [16] 21] have proposed algorithms, such as S-MAC, T-MAC, and D-MAC, that schedule the activities of all sensors in order to reduce the energy consumption. This paper bridges the gap, addressing in conjunction both data aggregation and an optimal duty cycle schedule, denotes as O-MAC, that centralized determines, for each sensor, when it wakes up and when it sleeps.

The third important energy consumption saving factor is dynamic power range. Carle et al. [6] discuss the tradeoff between power consumption and coverage of relay node. The power consumption of transmitting data is measured as $e_{u}\left(r_{u}\right)=r_{u}^{\alpha}+c$, where $\alpha$ is a signal attenuation constant (between 2 to 4 ) [13] 20] [22], $r_{u}$ is power range of the node $u$. Thus, the shorter range is used, the energy consumption is decreased.

The fourth factor is collisions avoidance that plays important roles in packet retransmissions as well as decreasing energy consumption. To reflect energy consumption by the number of retransmissions, the pure ALOHA approximation method [19], the extended Bianchi's model [5], and another previous work [13], based on the analysis in 18, were adopted to derive the expected number of retransmissions of a sender. In this paper, we include the equation in [13] as the node-to-node retransmission constraint.

In order to optimally solve the problem as we have stated it, we have formulated a mathematical model by which a data aggregation tree is constructed and the activities of all sensors are scheduled with adjustable radii and collision avoidance so as to minimize the total energy consumption. The solution to our mathematical formulation, where the objective function minimizes the total energy consumption of all sensors, subject to data aggregation, duty cycle scheduling, adjustable radii and the number of retransmissions constraints, is based on Lagrangian Relaxation (LR) in conjunction with optimization-based heuristics 9].

The remainder of this paper is organized as follows. In Section 2, a mixed integer nonlinear programming problem formulation of data aggregation routing problem with schedule assignment is proposed. In Section 3, LR-based approaches are presented. In Section 4, the heuristics for calculating good primal feasible solutions to these problems are developed. In Section 5, the computational results are reported. Finally, in Section 6 we present our conclusions.

\section{Problem Formulation}

A WSN is modeled as a graph of connected nodes, $G(V, L)$, where $V$ represents the nodes distributed on a two-dimensional plane (X_AXIS,Y_AXIS) and $(u, v) \in$ $L$ denotes links such that node $v$ can receive transmissions signal from node $u$. The problem is formulated as the followings. 
The objective function (1) is an expression of total energy consumption, including all facets of consumption within the network that take place when data is received or sent, or when nodes are idle. Note that rates $E_{r}$ of energy consumption are similar, whether receiving data or idle [17.

$$
Z_{I P}=\min \sum_{u \in V}\left[\left(m_{u}-n_{u}\right) E_{r}+\left(t_{d a t a}+R T S \sum_{v \in V} c_{u v}\right) e_{u}\left(r_{u}\right)\right]
$$

where $E_{r}$ denotes as energy consumption rate of a receiving or idle node; $E_{s}$ denotes as energy consumption rate of a transmission node; and $t_{d a t a}$ denotes as transmission time for transmitting a data packet, subject to:

- Path constraints: Constraint (2) shows the decision variable $x_{p}=1$ denoting that path $p \in P_{s}$, where $s$ belong to the set of source nodes $S$ and $P_{s}$ is the set of candidate paths from source $s$ to the sink node $\kappa$; otherwise, if $x_{p}=0$, no path $p$ is used. In order to realize constraints that determine the tree, original/destination (OD) pairs must on only one path. Thus, the equation is shown as (3).

$$
\begin{gathered}
x_{p}=0 \text { or } 1, \quad \forall p \in P_{s} \quad s \in S \\
\sum_{p \in P_{s}} x_{p} \leq 1, \quad \forall s \in S
\end{gathered}
$$

Once the path, $p$, is selected and the link $(u, v)$ is on the path, then the decision variable $y_{u v}$ must be set to 1 . This constraint is described by (4).

$$
\sum_{p \in P_{s}} x_{p} \delta_{p(u v)} \leq y_{u v}, \quad \forall s \in S \quad u, v \in V
$$

where $\delta_{p(u v)}$ is the indicator function: equal to 1 if link $(u, v)$ is on path $p$; equal to 0 otherwise. Thus, when the path $p$ is selected and link $(u, v)$ is on the path, the value of $x_{p} \delta_{p(u v)}$ is 1 and $y_{u v}$ must be set to 1 .

- Link constraints: Since this problem is to find a reversed multicast tree, five link constraints, Constraints (5)-(9), to requisite to describing structure of the tree.

1. Decision variable $y_{u v}=1$ denotes link $(u, v)$ is selected, whereas $y_{u v}=0$, link $(u, v)$ is not selected, shown as $(5)$. The source node $s$ must select one node to send its message to, meaning that the number of out-degree links must be 1 , shown as (6).

$$
\begin{gathered}
y_{u v}=0 \text { or } 1, \quad \forall u, v \in V \\
\sum_{v \in V} y_{s v}=1, \quad \forall s \in S
\end{gathered}
$$

2. The number of out-degree links of each node can be no greater than 1 , shown as (7).

$$
\sum_{v \in V} y_{u v} \leq 1, \quad \forall v \in V
$$


3. At least one relay node must be able to provide coverage to the sink node $\kappa$ for the message to be delivered, so the summation of in-degree links is at least 1 , shown as (8).

$$
\sum_{u \in V} y_{u \kappa} \geq 1
$$

4. The total number of links on the multicast tree must be at least the number of hops $H$ or the number of nodes in set $|S|$, whichever is greater 22], shown as $(9)$.

$$
\sum_{u \in V} \sum_{v \in V} y_{u v} \geq \max \{H,|S|\}
$$

- Node-to-node communication time constraints: Equation (10), which refers to [18, denotes the time $l_{u v}$ needed to transmit a packet from node $u$ to node $v$ by CSMA/CA protocol [3].

$$
\begin{array}{r}
l_{u v}=\frac{\left(e^{-\lambda \sum_{j \in V} z_{j u}(D I F S)}(R T S+S I F S+C T S+\bar{B})+D I F S+\bar{N}\right)}{e^{-\lambda \sum_{j \in V} z_{j u}(D I F S)} e^{-(R T S+S I F S+2 \theta) \sum_{j \in V} z_{j v}}}-\bar{N}, \\
\forall u, v \in V
\end{array}
$$

where the bound of the delay of each link is described as (11).

$$
R T S+S I F S+C T S+\bar{B}+D I F S \leq l_{u v} \leq M_{5}, \quad \forall u, v \in V
$$

Note that $M_{5}$ denotes as the maximum node-to-node successful transmission time; $\lambda$ denotes as the arrival rate of an event occurrence; $\theta$ denotes as the propagation time to send a packet; $\bar{B}$ denotes as average random backoff time; $\bar{N}$ denotes as average Network Allocation Vector (NAV) time; DIFS denotes as Distributed Inter-Frame Space; SIFS denotes as Short InterFrame Space; $R T S$ denotes as RTS transmission time; and $C T S$ denotes as CTS transmission time.

The decision variable, $z_{u v}$, is a $0-1$ variable, shown as (12). It is equal to 1 when node $v$ is within the transmission range of node $u$ and link $(u, v)$ is selected, shown as (13). However, $z_{u v}$ must be equal to 0 as node $u$ does not need to transmission any data, shown as (14); otherwise the equation is violated.

$$
\begin{gathered}
z_{u v}=0 \text { or } 1, \quad \forall u, v \in V \\
\frac{r_{u}-d_{u v}}{M_{1}}+\left(1-y_{u v}\right) \leq z_{u v}, \quad \forall u, v \in V \\
z_{u v} d_{u v} \leq r_{u}, \quad \forall u, v \in V
\end{gathered}
$$

where $d_{u v}$ denotes as Euclidean distance between the node $u$ and the node $v$. 
- The number of retransmissions: As described in Section 1, the number of retransmissions is calculated by (15) and (16), which the right hand side of (15) is referred to [18 to calculate the expected retransmissions, $c_{u v}$, and then set the value to be an integer.

$$
\begin{gathered}
c_{u v} \geq \frac{e^{-\left(1-y_{u v}\right) M}}{e^{-\lambda(R T S+S I F S+2 \theta) \sum_{j \in V} z_{j v}}}, \quad \forall u, v \in V \\
c_{u v} \in\{0,1,2, \ldots, T\}, \quad \forall u, v \in V
\end{gathered}
$$

- Scheduling constraints: Constraint (17) puts limits on the time at which all incoming flow from nodes to a node $u$ must be aggregated, denotes as $m_{u}$. Note that $M_{3}$, which is the longest end-to-end delay of the network, denotes the upper bound of $m_{u}$, which described as (18).

$$
\left(m_{v}+l_{u v}+\varepsilon\right)-M_{3}\left(1-y_{v u}\right) \leq m_{u}, \quad \forall u, v \in V
$$

where $\varepsilon$ is estimation error value, which is used for time synchronized error.

$$
0 \leq m_{u} \leq M_{3}, \quad \forall u \in V
$$

A node $u$ involved in an aggregation tree is subject to (19). The wake up time of node $u$ must be earlier than the aggregation time of nodes that receive from it, denotes as $n_{u}$. Note that $M_{4}$, which is the longest end-to-end delay of the network, denotes the upper bound of $n_{u}$, which described as (20).

$$
\begin{gathered}
n_{u} \leq m_{u}+M_{4}\left(1-y_{v u}\right), \quad \forall u, v \in V \\
0 \leq n_{u} \leq M_{4}, \quad \forall u \in V
\end{gathered}
$$

\section{Solution Approach}

The LR-based approach 9] is a flexible solution strategy that permits us to exploit the fundamental structure of possible optimization problems by relaxing complicated constraints into the objective function with Lagrangian multipliers [1] 9]. Before executing the LR procedures, Constraint (10) is transformed to be approximated function with the error is estimated less than $5 \%$. The natural logarithm of either side renders this function solvable as:

$$
\begin{aligned}
\ln \left(l_{u v}\right)= & \ln (R T S+S I F S+C T S+330)+0.115+ \\
& 0.017 \sum_{j \in V} z_{j v}+\lambda(R T S+S I F S+2 \theta) \sum_{j \in V} z_{j v}
\end{aligned}
$$

For Constraint (15), we also take natural logarithm on both sides and get:

$$
\ln \left(c_{u v}\right) \geq \lambda(R T S+S I F S+2 \theta) \sum_{j \in V} z_{j v}-M_{5}\left(1-y_{u v}\right)
$$


Accordingly, the primal optimization problem is transformed into a Lagrangian dual problem. The relaxation of (4), (13), (14), (17), (19), (21), and (22) transforms the objective function (1) into a Lagrangian dual problem with a vector of non-negative Lagrangian multipliers (i.e., $\mu_{s u v}^{1}, \mu_{u v}^{2}, \mu_{u v}^{3}, \mu_{u v}^{4}, \mu_{u v}^{5}, \mu_{v u}^{6}$ and $\mu_{u v}^{7}$ ).

$$
\begin{aligned}
& Z_{L R}\left(\mu_{s u v}^{1}, \mu_{u v}^{2}, \mu_{u v}^{3}, \mu_{u v}^{4}, \mu_{u v}^{5}, \mu_{v u}^{6}, \mu_{u v}^{7}\right)= \\
& \min \sum_{u \in V}\left[\left(m_{u}-n_{u}\right) E_{r}+\left(t_{d a t a}+R T S \sum_{v \in V} c_{u v}\right) e_{u}\left(r_{u}\right)\right]+ \\
& \sum_{s \in S} \sum_{u \in V} \sum_{v \in V} \mu_{s u v}^{1}\left(\sum_{p \in P_{s}} x_{p} \delta_{p(u v)}-y_{u v}\right)+ \\
& \sum_{u \in V} \sum_{v \in V} \mu_{u v}^{2}\left(\frac{r_{u}-d_{u v}}{M_{1}}+\left(1-y_{u v}\right)-z_{u v}\right)+ \\
& \sum_{u \in V} \sum_{v \in V} \mu_{u v}^{3}\left(z_{u v} d_{u v}-r_{u}\right)+ \\
& \sum_{u \in V} \sum_{v \in V} \mu_{u v}^{4}\left(\lambda(R T S+S I F S+2 \theta) \sum_{j \in V} z_{j v}-M_{5}\left(1-y_{u v}\right)-\ln \left(c_{u v}\right)\right)+ \\
& \sum_{u \in V} \sum_{v \in V} \mu_{u v}^{5}\left(\begin{array}{l}
\ln (R T S+S I F S+C T S+330)+0.115+0.017 \sum_{j \in V} z_{j u} \\
+\lambda(R T S+S I F S+2 \theta) \sum_{j \in V} z_{j v}-\ln \left(l_{u v}\right)
\end{array}\right)+ \\
& \sum_{v \in V} \sum_{u \in V} \mu_{v u}^{6}\left(m_{v}+l_{v u}+\varepsilon-M_{3}\left(1-y_{v u}\right)-m_{u}\right)+ \\
& \sum_{u \in V} \sum_{v \in V} \mu_{u v}^{7}\left(n_{u}-m_{v}-M_{4}\left(1-y_{u v}\right)\right)
\end{aligned}
$$

subject to: (2), (3), (5), (6), (7), (8), (9), (11), (12), (16), (18) and (20).

Accordingly, the (LR) is decomposed into seven independent and solvable subproblems, (SUB1), (SUB2), (SUB3), (SUB4), (SUB5), (SUB6), and (SUB7). Based on the weak Lagrangian duality theorem, which holds that for any given set of nonnegative multipliers, the LR approach finds the lower bound of the value of the primal objective value [1], in this case, $Z_{D}$ is a lower bound on $Z_{I P}[$

\section{Primal Feasible Solution}

To construct a reversed multicast tree, the decision variable $x_{p}$ is our choice for finding primal feasible solutions, in view of the fact that once $x_{p}$ is determined, the other decision variables are also determined. We have developed a heuristic for routing policy adjustment based on $x_{p}$. First, to ensure that each OD pair perceives the same link weight for the same link, we make adjustments to $c_{u v}=\left(\sum_{s \in S} \mu_{s u v}^{1}+\mu_{u v}^{2}+\mu_{u v}^{3}+\mu_{u v}^{4}+\mu_{v u}^{6}+\mu_{u v}^{7}\right) d_{u v}^{2}$. The transmission graph will be a reversed multicast tree, meeting the requirements of (2)-(9). Next, the solution set of $x_{p}$ is generated by the proposed LR-based heuristic. The procedures are shown as follows for obtaining a primal feasible solution (Primal_Heuristic_Algorithm ()$)$ that solves the problem. Accordingly, the LR-based algorithm for solving primal problem is used according to the procedures in 1 or 9.

\footnotetext{
${ }^{1}$ For more information, please contact with the correspondence author.
} 
$\overline{\text { Step 1. Initially, } c_{u v}=\left(\sum_{s \in S} \mu_{s u v}^{1}+\mu_{u v}^{2}+\mu_{u v}^{3}+\mu_{u v}^{4}+\mu_{v u}^{6}+\mu_{u v}^{7}\right) d_{u v}^{2}}$; pseudo links connect the source nodes to the pseudo source; and a pseudo link between the sink node and pseudo destination are also set. The weights of these pseudo links are 0 .

Step 2. The Dijkstra's algorithm is used to find the shortest path from the pseudo source $O$ to the pseudo destination $D$.

Step 3. Once the path has been determined, the nodes on the path are marked and pseudo links with weight 0 are added between these marked nodes and the pseudo destination. The weight of pseudo link, which is on the path between pseudo source and its next hop, is set to a infinite.

Step 4. Steps 2-3 are repeated until all source nodes are marked.

Step 5. Now we have a reversed multicast tree after cyclic path has been checked. Once the $\left\{x_{p}\right\}$ is determined, $\left\{y_{u v}\right\}$ is also determined. According to the selected link set $\left\{y_{u v}\right\}$, the power range $r_{u}$, the number of nodes within the node $u$ 's transmission area $z_{u v}$, and link transmission time $l_{u v}$ to successful transmission are calculated.

Step 6. The earliest wake up time and latest aggregated time are calculated along the DFS (Depth First Search) traced nodes. Once $m_{u}$ and $n_{u}$ are determined, the duty cycle schedule of each sensor node $u$ is determined. The total energy consumption per cycle is calculated.

Figure 1 shows an example of the proposed routing algorithm. The pseudo source $O$, pseudo destination $D$, and pseudo links $O 1, D 5, D 6, D 7$, and $D 8$ with weight 0 are initialized. In first iteration, we find the path $O-7-4-2-1-D$ from $O$ to $D$. The nodes on the paper are marked in the tree set $T$. Then the pseudo links $D 2, D 4$, and $D 7$ with weight 0 are added. But the weight of pseudo

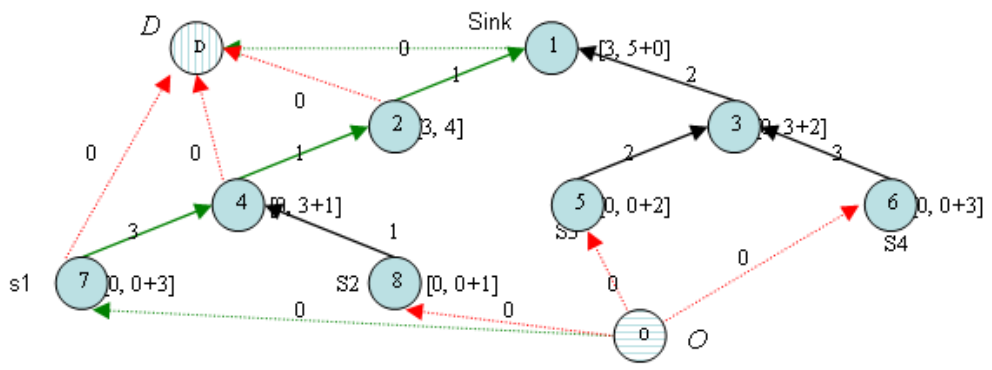

Fig. 1. The pseudo source $O$ and pseudo destination $D$ are added. The pseudo links are added to connect source nodes and sink node, respectively. The value on the link signifies the delay to successfully send data to next node; the $\left[n_{u}, m_{u}+l_{u v}\right]$ of each node denotes the earliest wake up time and the complete aggregated time for successful transmission. 
link $O 7$ is set to be infinite. Iteration by iteration, additions to the reversed multicast tree are made until all source nodes in the network have been marked in the tree.

\section{Evaluation and Experimental Results}

The experimental networks were comprised of $N$ sensor nodes, with 150 nodes in the example given in Figs. 4 and network with various numbers of nodes, up to 250, in Figs. 2 and 3 randomly placed in a $10 * 10$ square unit area. The relative experimental parameter settings are referred to 3] and [13. To evaluate the solution quality of our proposed algorithm, we compared it with three existing data aggregation routing algorithms: the GIT, SPT, and CNS algorithms are proposed in [12. We also compared it with exist schedule strategies, such as S-MAC and T-MAC. The experimental results are shown in Fig. 4.

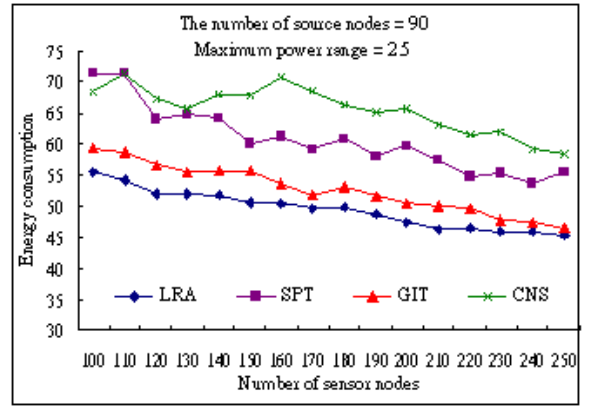

Fig. 2. Network sizes experimental results

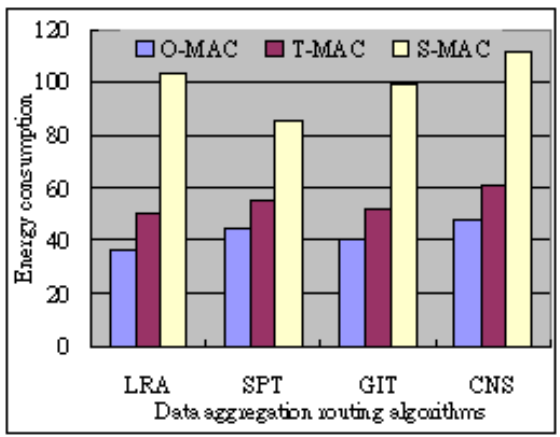

(a) the number of sources node is 90

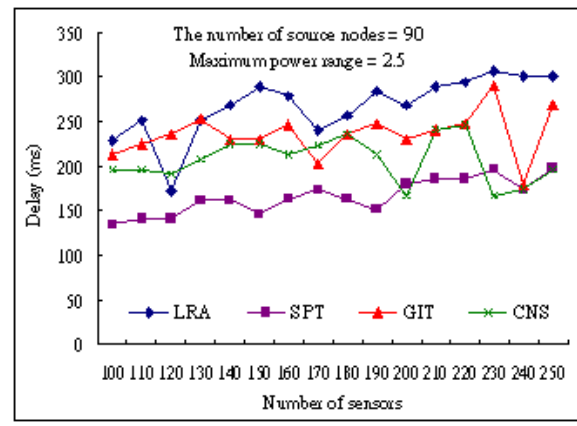

Fig. 3. Maximum end-to-end delay experimental results

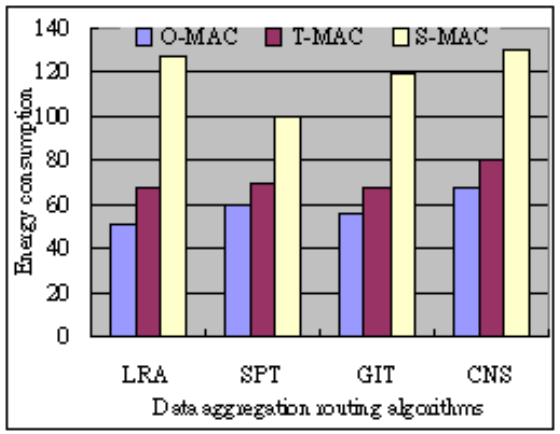

(b) the number of sources node is 50

Fig. 4. The total energy consumption by combining data aggregation routing heuristics and scheduling heuristics 
Figure 2 depicts the experimental results by which we assess the quality of each heuristics as used in networks of differing sizes, each with a fixed number of sources but a adjustable radii. The consumption decreases with the shorter radius when energy consumption evaluated by exponential distance. Thus, when there is increased network sizes, the density of sensor nodes in the fixed deployment area is higher, and overall energy consumption decreases. The effect of this on our proposed algorithm is minimal, so the solution quality of the LRA algorithm is superior to the other heuristics by up to $28.8 \%$.

However, a longer delay does arise with the proposed algorithm than that from other algorithms, shown as Fig. 3. This additional delay, the cost of reducing duplicate transmissions, arises due to the time necessary to aggregate data before forwarding it. Other algorithms, such as SPT, do receive the most up-to-date information from the sensor nodes by adopting a single pair and a shortest path heuristic, but it must be said that the energy consumption of a network that implements one of these other algorithms is significantly higher than that achieved by the proposed algorithm.

Figure 4 shows the scheduling algorithms combine with the above data aggregation routing algorithms. As our exceptive, the proposed O-MAC adopt the variable awake up duty cycle according the data aggregation tree outperforms than other S-MAC (with fixed duty cycle) and T-MAC (variable duty cycle by timeout) up to $27.6 \%$ and $65 \%$. The reason is S-MAC take much time on idle listening. Even T-MAC enhances from the S-MAC by timeout mechanism, it takes addition timeout on idle listening than the proposed algorithm.

\section{Conclusions}

The energy efficiency of WSNs can be improved by data aggregation routing, the reduction of idle listening, adjustable radii, and collision avoidance. To address these considerations, we propose a mixed integer nonlinear mathematical formulation of duty cycle scheduling with data-aggregation routing. This paper presents a LRA algorithm that is derived from the LR approach, making possible the construction of an energy-efficient data aggregation tree that takes into consideration scheduling of transmission activities and tradeoffs between data aggregation, adjustable radii, and collision avoidance. According to the experimental results, the proposed LRA algorithm outperforms other heuristics in tests, especially in large networks 3. More specifically, our proposed scheduling heuristic improves the energy conservation, which it does up to $65 \%$ over S-MAC.

\section{References}

1. Ahuja, R.K., Magnanti, T.L., and Orlin, J.B.: Network Flows: Theory, Algorithms, and Applications. Ch. 16 Prentice-Hall (1993)

2. Akyildiz, I.F., Su, W., Sankarasubramaniam, Y., Cayirci E.: A Survey on Sensor Networks. IEEE Communications Magazine 40(8) (2002) 102-114 
3. ANSI/IEEE: 802.11: Wireless LAN Medium Access Control (MAC) and Physical Layer (PHY) Specifications. IEEE Standard 802.11 (2000)

4. Bhardwaj, M., Chandrakasan, A.P.: Bounding the Lifetime of Sensor Networks via Optimal Role Assignments. Proc. IEEE INFOCOM, New York NY (2002) 15871596

5. Bianchi, G.: Performance Analysis of the IEEE 802.11 Distributed Coordination Function. IEEE Journal on Selected Areas in Communications 18(2) (2000) 535547

6. Carle, J., Simplot, D.: Energy Efficient Area Monitoring by Sensor Networks. IEEE Computer 37(2) (2004) 40-46

7. Chang, J.H., Tassiulas, L.: Maximum Lifetime Routing in Wireless Sensor Networks. IEEE/ACM Transactions on Networking (TON) 12(4) (2004) 609-619

8. Dam, T.V., Langendoen K.: An Adaptive Energy-Efficient MAC Protocol for Wireless Sensor Networks. Proc. ACM SenSys Los Angeles (2003) 171-180

9. Fisher M.L.: The Lagrangian Relaxation Method for Solving Integer Programming Problems. Management Science 27(1) (1981) 1-18

10. Garey, M.R., Johnson, D.S.: Computers and Intractability: A Guide to the Theory of NP-completeness Freeman San Francisco (1979)

11. Kalpakis, K., Dasgupta, K. Namjoshi, P.: Efficient Algorithms for Maximum Lifetime Data Gathering and Aggregation in Wireless Sensor Networks. Computer Networks Journal 42(6) (2003) 697-716

12. Krishnamachari, B., Estrin, D., Wicker, S.: Modeling Data-Centric Routing in Wireless Sensor Networks. USC Computer Engineering Technical Report CENG (2002)

13. Lin, F.Y.S., Yen, H.H. Lin, S.P.: MAC Aware Energy-Efficient Data-Centric Routing in Wireless Sensor Networks. Proc. IEEE ICC Istanbul Turkey (2006)

14. Liu, H., Wan, P.J., Yi, C.W., Jia, X., Makki, S., Pissinou, N.: Maximal Lifetime Scheduling in Sensor Surveillance Networks. Proc. INFOCOM Miami (2005) 24822491

15. Lu, G., Krishnamachari, B., Raghavendra, C.: An Adaptive Energy-Efficient and Low-Latency MAC for Data Gathering in Sensor Networks. Proc. WMAN (2004)

16. Lu, G., Sadagopan, N., Krishnamachari, B., and Goel, A.: Delay Efficient Sleep Scheduling In Wireless Sensor Network. Proc. INFOCOM Miami Florida USA (2005) 2470-2481

17. Stemm, M., Katz, R.H., Measuring and Reducing Energy Consumption of Network Interfaces in Hand-held Devices. IEICE Transactions on Communications E80B(8) (1997) 1125-1131

18. Sheu, S.T., Tsai, T.H. Chen, J.H.: MR2RP: The Multi-Rate and Multi-Range Routing Protocol for IEEE 802.11 Wireless Ad Hoc Networks. ACM/Kluwer Wireless Networks 9(8) (2003) 165-177

19. Shiou, C.W., Lin, F.Y.S., Cheng, H.C., Wen, Y.F.: Optimal Energy-Efficient Routing for Wireless Sensor Networks. Proc. IEEE AINA Taipei Taiwan (2005) 325-330

20. Wieselthier, J.E., Nguyen, G.D., Ephremides, and A.: Energy-Efficient Broadcast and. Multicast Trees in Wireless Networks. Mobile Networks and Applications (MONET) 7(2) (2002) 481-492

21. Ye, W., Heidemann, J., Estrin, D.: An Energy-efficient MAC Protocol for Wireless Sensor Networks. Proc. IEEE INFOCOM New York, NY (2002) 1567-1576

22. Yen, H.H., Lin, F.Y.S. and Lin, S.P.: Energy-Efficient Data-Centric Routing in Wireless Sensor Networks. IEICE Trans. on Communications E88-B(16) (2005) 4470-4480 